\title{
Cooper pair cotunneling in single charge transistors with dissipative electromagnetic environment
}

\author{
S. V. Lotkhov, S. A. Bogoslovsky, A. B. Zorin and J. Niemeyer \\ Physikalisch-Technische Bundesanstalt, Bundesallee 100, 38116 Braunschweig, Germany
}

(Dated: November 21, 2018)

\begin{abstract}
We observed current-voltage characteristics of superconducting single charge transistors with onchip resistors of $R \sim R_{Q}=h / 4 e^{2} \approx 6.45 \mathrm{k} \Omega$, which are explained in terms of Cooper-pair cotunneling. Both the effective strength of Josephson coupling and the cotunneling current are modulated by the gate-induced charge on the transistor island. For increasing values of the resistance $R$ we found the Cooper pair current at small transport voltages to be dramatically suppressed.

PACS numbers: 74.50.+r, 74.25.Fy, 74.40.+k
\end{abstract}

During the last decade superconducting circuits with mesoscopic tunnel junctions have attracted much attention of researchers. Remarkable quantum properties of these circuits, which stem from interaction between Josephson and charging effects, enable the construction of charge-based qubits [1], sensitive electrometers [2], amplifiers [3], etc. Since the Josephson supercurrent itself is of an elastic nature, tunneling of Cooper pairs in such circuits is dramatically influenced by the Coulomb interaction and dissipation.

The tunneling in the two-junction system (single Cooper pair transistor) with a dissipative environment was recently theoretically studied by Wilhelm et al. 4] and experimentally investigated by Kycia et al. [5] and Lu et al. [6] who used the advantage of locally tunable dissipation associated with the conductance of 2DEG beneath the transistor structure. These papers were focused on the regime of sequential tunneling of Cooper pairs (STCP) dominating when the electric potential of the transistor island was tuned "in resonance" (see parameter diagram in Fig. 3 of Ref. [4]). Although a quantum regime of simultaneous pair tunneling across both junctions, i.e. cotunneling of Cooper pairs (CTCP), was roughly evaluated in Ref. [4], it had not been, to our knowledge, studied experimentally.

Under the term "cotunneling" we understand discrete tunneling of single Cooper pairs across a whole transistor [7]. Although numerous experiments show that Cooper pair transistors without deliberately attached resistors exhibit either a gate-dependent switching current at ramp-current bias or a current peak at low voltage bias (see, e.g., Ref. [8]), these data indicate to a through supercurrent. Due to unavoidable dissipation leading to fluctuations of the overall Josephson phase $\phi$, the observable value of the supercurrent is non-zero at finite transport voltage. Phase $\phi$ is still a good variable, while the number of transferred Cooper pairs is meaningless, so the term "single" is not applicable to this regime.

When an appreciable impedance is introduced into the network, i.e. $\operatorname{Re} Z=R \sim R_{Q}=h / 4 e^{2} \approx 6.45 \mathrm{k} \Omega$, quantum fluctuations of phase $\phi$ increase dramatically, and this leads to incoherent tunneling of individual pairs across the transistor, i.e. CTCP. In this paper we investigate the CTCP effect in $\mathrm{Al}$ transistors with thin-film $\mathrm{Cr}$ local resistors of different values $R$ inserted into the bias leads. We observe the gate and bias voltage dependencies of CTCP current and demonstrate that a significant suppression of CTCP at small bias voltage can be achieved by a further increase in the resistance $R$.

The Cooper pair transistors were fabricated using the angle evaporation technique for three metal shadows (Cr/Al-1(oxidized)/Al-2) evaporated at different angles through the same mask. Details of the fabrication process can be found elsewhere [9]. We put on the same chip a number of nominally identical symmetrical transistors (with tunnel junctions of type $\mathrm{Al} / \mathrm{AlO}_{\mathrm{x}} / \mathrm{Al}$ with dimensions $40 \mathrm{~nm} \times 80 \mathrm{~nm}$ ) differing only in the lengths of the Cr microstrips (each $0.1 \mu \mathrm{m}$ - wide and $7 \mathrm{~nm}$ - thick), ranging from $0.32 \mu \mathrm{m}$ to $3 \mu \mathrm{m}$. The specific film resistivity was from $5.5-7 \mathrm{k} \Omega$ per $\mu \mathrm{m}$ of length yielding values of resistance $R$ in the range of $2-20 \mathrm{k} \Omega$.

The measurements were performed in the dilution refrigerator at the base temperature of about $20 \mathrm{mK}$. The sample was mounted in a copper shielding box. All bias and gate lines were supplied with microwave filters, made of Thermocoax ${ }^{\circledR}$ cable located in the coldest part of the setup. The electric diagram of the sample bias is shown in the upper inset on Fig. 1. The normal-state tunnel resistance of the transistors, $R_{\mathrm{tr}}=R_{1}+R_{2}$, where $R_{1,2}$ are tunnel resistances of individual junctions, was remarkably uniform and ranged from $40 \mathrm{k} \Omega$ to $44 \mathrm{k} \Omega$. The superconducting gap and the corresponding values of the Ambegaokar-Baratoff critical current of the junctions (assuming symmetry of the transistors) were estimated to be $\Delta_{\mathrm{Al}} \approx 200 \mu \mathrm{eV}$ and $I_{c}^{0} \approx 16 \mathrm{nA}$, respectively. These data yield a characteristic Josephson energy of junctions $E_{J 1, J 2}=E_{J} \equiv \frac{\Phi_{0}}{2 \pi} I_{c}^{0} \approx 30 \mu \mathrm{eV}$, where $\Phi_{0}=\frac{h}{2 e}$ is the flux quantum. From the normal-state $I-V$ curves we evaluated a characteristic charging energy of the transistor island $E_{c} \equiv e^{2} / 2 C_{\Sigma} \approx 150-160 \mu \mathrm{eV}$, where $C_{\Sigma}=C_{1}+C_{2}+C_{g} \approx 530 \mathrm{aF}$ (typical value) is the total capacitance of the island and $C_{1,2}=C \approx 260 \mathrm{aF}$ and $C_{g} \approx 9$ aF $\ll C$ are the capacitances of junctions and gate, respectively. Due to the small $C_{g}$, the gate- 
induced charge is fixed, $Q_{0}=C_{g} V_{g}+Q_{00}$, where $Q_{00}$ is the background charge. The reasonably small ratio of characteristic energies in our samples, $\lambda_{0}=E_{J} / E_{c} \approx 0.2$, allowed us both clear observation and transparent interpretation of the CTCP effect $\left(\propto \lambda_{0}^{4}\right)$. Moreover, linearity and small resistor lengths let us consider them as lumped elements (pure resistors) and neglect in our model their distributed self-capacitance $(\approx 60 \mathrm{aF} / \mu \mathrm{m})$.

$I-V$ characteristics of superconducting single charge transistors are generally featured by several contributions to the net current. These contributions result from the tunneling of Cooper pairs, single quasiparticles and combining tunneling of both pairs and quasiparticles. At small transport voltage and temperature $\left(e V, k_{B} T \ll 2 E_{c}, 2 \Delta_{\mathrm{Al}}\right)$, the tunneling of quasiparticles cannot make a notable contribution to the net current because of the exponentially large subgap resistance. In this limit, the Cooper pair (Josephson) current component is dominant. Still, the rare events of quasiparticle tunneling (poisoning of the island) at $\left|Q_{0}\right| \geq e / 2$ were possible and they resulted in 1e-periodic gate dependence of the $I-V$ curves (see bottom inset in Fig. 1). Therefore, slow ramping of the gate voltage (when measuring gate-modulation curves) provided cyclic variations of the polarization charge on the island $Q_{0}$ in the range from $-e / 2$ to $e / 2$. Within this range of $Q_{0}$, change of the island charge by $2 \mathrm{e}$ would require an energy $\delta E_{\mathrm{ch}}$ ranging between $2 E_{c}$ (at $\left.Q_{0}= \pm e / 2\right)$ and $4 E_{c}$ (at $Q_{0}=0$ ). Therefore, the process of Cooper pair transfer with "landing" on the island, i.e. the STCP, was exponentially suppressed, $\propto \exp \left(-\delta E_{\mathrm{ch}} / k_{B} T\right)$, so only the CTCP contributed to the net current.

Figure 1 shows the $I-V$ curves of three samples with resistance $R$ of nominally zero value (the actual impedance in this case is of the order of the vacuum impedance $Z_{0} \approx 377 \Omega$, although its frequency dependence is unknown), $3 \mathrm{k} \Omega$ and $5 \mathrm{k} \Omega$. One can see a remarkable change from the peak (cf. Fig. 1 in Ref. [8]) to step-like and then to an almost linear shape of the $I-V$ curve that can be interpreted as a growth of fluctuations of the total Josephson phase leading to a depression of the supercurrent feature.

A similar behavior of a single junction was described by Averin et al. [10] in terms of incoherent tunneling of pairs yielding current $I=2 e[\Gamma(2 e V)-\Gamma(-2 e V)]$ and a tunneling rate

$$
\Gamma(E)=\frac{\pi}{2 \hbar} E_{J}^{2} P(E),
$$

where the function $P(E)$ with $E=2 e V$ describes the probability of energy dissipation in the environment network. The experimental observation of this regime was reported by Kuzmin et al. 11.

In the case of a transistor, one may consider $E_{J}$ in Eq. (1) as an effective Josephson coupling dependent on the gate voltage $V_{g}$. To support this idea, we observed

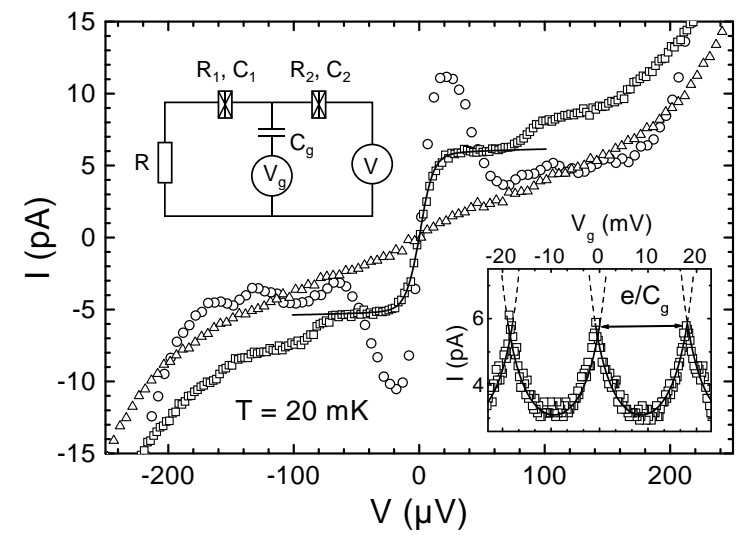

FIG. 1: The electric circuit diagram (upper inset) and $I-V$ curves of three Cooper pair transistors with different nominal values of the resistance $R$ (circles - 0 , squares $-3 \mathrm{k} \Omega$ and triangles $-5 \mathrm{k} \Omega$ ) at gate voltage values $V_{g}$ corresponding to maximum of current. The solid line shows computed data assuming a symmetric transistor with $E_{J}=26 \mu \mathrm{eV}$ and a resistor of $R=3.3 \mathrm{k} \Omega$ with an effective temperature of $90 \mathrm{mK}$. The gate dependence in the transistor with $R=3 \mathrm{k} \Omega$ at $V=30 \mu \mathrm{V}$ is presented in the bottom inset, where the solid (dashed) line shows the data calculated within an experimentally accessible (inaccessible) range.

the shapes of the $I-V$ curves similar to those given by the $P$-theory for single junctions with ohmic environment [10, 12]. Moreover, the observed shapes were immutable to $V_{g}$ whose variation led only to scaling of the current.

To model the behavior of our $I-V$ curves, we present the Josephson coupling term of the transistor Hamiltonian, $H_{J}=-E_{J 1} \cos \varphi_{1}-E_{J 2} \cos \varphi_{2}$, in the form

$$
H_{J}(\phi, \varphi)=-E_{J}^{\operatorname{tr}}(\phi) \cos [\varphi+\gamma(\phi)],
$$

where $E_{J}^{\operatorname{tr}}=\left(E_{J 1}^{2}+E_{J 2}^{2}+2 E_{J 1} E_{J 2} \cos \phi\right)^{1 / 2}, \tan \gamma=$ $\left[\left(E_{J 1}-E_{J 2}\right) /\left(E_{J 1}+E_{J 2}\right)\right] \tan (\phi / 2)$ and $\phi=\varphi_{1}+\varphi_{2}$ ( $\varphi_{i}$ is the phase drop across the $i$-th junction). The difference phase $\varphi=\frac{1}{2}\left(\varphi_{1}-\varphi_{2}\right)$ is a variable conjugate to the island charge $Q=-2 e i \frac{\partial}{\partial \varphi}$. $Q$ enters the charging energy term $H_{\mathrm{ch}}(Q)=E_{c}\left[\left(Q+Q_{0}\right) / e\right]^{2}$. For a given value of operator $\phi$, the transistor Hamiltonian $H_{\mathrm{tr}}=H_{\mathrm{ch}}(Q)+H_{J}(\phi, \varphi)$ is similar to that of a single junction. Taking the fact into account that the commutator $\left[\phi, H_{\mathrm{tr}}\right]=0$, we can diagonalize $H_{\mathrm{tr}}(\phi, \varphi, Q)=$ $H_{\mathrm{tr}}(\phi, q, n)$ in the basis of the Bloch states $|q, n\rangle$, where $q$ is the quasicharge and $n=0,1,2, \ldots$ the band index [13]. Assuming that only the ground state, $n=0$, is occupied (i.e., the effects of thermal, photon-assisted and Zener breakthrough excitations of higher bands are negligibly small) and noting that variable $q$ is decoupled from environmental degrees of freedom due to small $C_{g}$ and fixed by $V_{g}$, we present the transistor Hamiltonian as

$$
H_{\mathrm{tr}}=E_{0}\left[\lambda(\phi), Q_{0}\right] .
$$


Here $E_{0}$ is the ground state energy of an equivalent single junction with variable ratio $\lambda=E_{J}^{\operatorname{tr}}(\phi) / E_{c} \leq 2 \lambda_{0}$ and $q=Q_{0} . \quad E_{0}$ is $2 \pi$-periodic function of $\phi$ and $2 e-$ periodic function of charge $Q_{0}$ and its shape depends on the relation between $E_{J 1, J 2}$ and $E_{c}$.

Due to the periodic dependence on $\phi$, the Hamiltonian Eq. (3) can be expanded into a Fourier series

$$
H_{\mathrm{tr}}\left(\phi, Q_{0}\right)=-\sum_{k=0}^{\infty} E_{J}^{(k)}\left(Q_{0}\right) \cos (k \phi) .
$$

For $Q_{0} \neq e \bmod (2 e)$ or/and at notable asymmetry of the transistor, $E_{J 1} \neq E_{J 2}$, the terms with $k=0$ and 1 are dominating in Eq. (44). This is in accordance with almost sinusoidal supercurrent-phase relation [14] with effective critical current $I_{c}^{\operatorname{tr}}\left(Q_{0}\right)=\frac{2 \pi}{\Phi_{0}} E_{J}^{(1)}\left(Q_{0}\right)$ modulated by gate (see corresponding plots for different values of $\lambda_{0}$ in Fig. 2a of Ref. 15]). In particular, in the symmetric transistor with $\lambda_{0} \ll 1, I_{c}^{\text {tr }}$ can be found explicitly from Eqs. (4)-(5) of Ref. 15] using the wave functions given by Eq. (A11) of Ref. [13], i.e.,

$$
I_{c}^{\operatorname{tr}}\left(Q_{0}\right)=\frac{\lambda_{0}}{4}\left[1-\left(Q_{0} / e\right)^{2}\right]^{-1} I_{c}^{0} .
$$

In the degenerate case of $Q_{0}=e \bmod (2 e)$ and $E_{J 1}=$ $E_{J 2}=E_{J} \lesssim E_{c}$, the ground state energy Eq. (3) is $E_{0} \approx$ $-E_{J}\left|\cos \frac{\phi}{2}\right|+$ const, while the maximum critical current is $I_{c}^{\operatorname{tr}}(e) \approx 0.5 I_{c 1, c 2}$. The Fourier coefficients in Eq.(4) are equal to

$$
E_{J}^{(k)}(e)=\frac{4}{\pi} \frac{(-1)^{k-1}}{(2 k)^{2}-1} E_{J}, \quad k \geq 1,
$$

so the amplitudes of the principal (first harmonic) term $E_{J}^{(1)}(e) \approx 0.42 E_{J}$ and the ratio $\left|E_{J}^{(2)}(e) / E_{J}^{(1)}(e)\right|=0.2$.

The expansion Eq. (4) presents the transistor Hamiltonian as a weighted sum of operators $e^{ \pm i k \phi}$, each describing tunneling of a cluster of $k$ Cooper pairs through the transistor in the positive (negative) direction. This presentation enables particular rates for tunneling of $k$ pairs, $\Gamma_{k}(E)$, to be found and the total current to be calculated via the corresponding sum.

Due to finite impedance $R$, the phase $\phi$ couples to the environmental degrees of freedom via an operator of phase on the resistor $\phi_{R}: \phi=\frac{2 \pi}{\Phi_{0}} V t-\phi_{R}$. Phase $\phi_{R}$ is related to the infinite set of bath variables. The effect of this coupling can be treated by means of function $P$ determined by the charge of the tunneling particle (cluster of particles), temperature and the real part of total impedance $Z$ as seen by the tunneling charge [16].

In our case, the real part of the impedance is equal to

$$
\operatorname{Re} Z(\omega)=R /\left[1+\left(\omega R C_{\mathrm{tr}}\right)^{2}\right]
$$

where the transistor capacitance $C_{\mathrm{tr}}=\frac{C_{1} C_{2}}{C_{1}+C_{2}} \approx \frac{C}{2}$ (corresponding to the characteristic charging energy of $4 E_{c}$ )

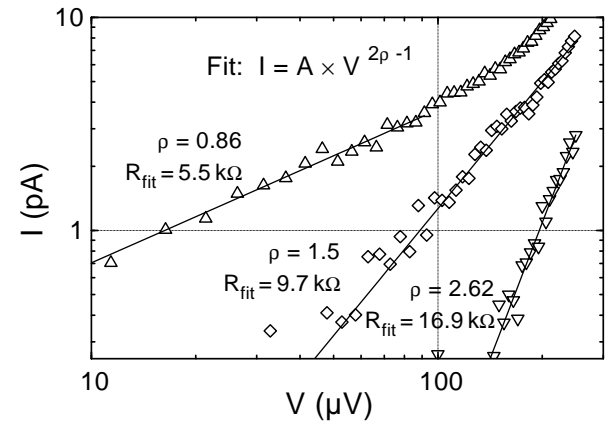

FIG. 2: The $I-V$ curves of three Cooper pair transistors with different values of resistance $R$ (up-triangles $-5 \mathrm{k} \Omega$, diamonds - $9 \mathrm{k} \Omega$ and down-triangles $-17 \mathrm{k} \Omega$, cf. the fit values $\left.R_{\mathrm{fit}}\right)$ and their power-function fit in accordance with Eq. (10). The fitted values of prefactor $A=I_{0} V_{0}^{1-2 \rho}$ were used to restore the values of $E_{J}$ ( $=33,34$ and $28 \mu \mathrm{eV}$, respectively).

and the tunneling charge is equal to $2 k e$. The rates, $\Gamma_{k}(E)=\frac{\pi}{2 \hbar}\left[E_{J}^{(k)}\right]^{2} P(E)$, and the total cotunneling current,

$$
I_{\mathrm{cot}}=2 e \sum_{k=1}^{\infty} k\left[\Gamma_{k}(2 k e V)-\Gamma_{k}(-2 k e V)\right]
$$

are found perturbatively, assuming $0.5 E_{J} / 4 E_{c}=\lambda_{0} / 8 \ll$ 1. If $Q_{0}$ not close to $e$, the term with $k=1$ is essential, and the cotunneling current is given by the formula similar to that derived by Ingold et al. [12] for tunneling across a single junction at $2 \mathrm{eV} \ll \hbar / R C$,

$$
\begin{aligned}
I_{\cot }= & \frac{\Phi_{0}\left[I_{c}^{\operatorname{tr}}\left(Q_{0}\right)\right]^{2}}{4 E_{c}} e^{-2 \gamma \rho} \rho^{2 \rho}\left(\frac{2 E_{c}}{\pi^{2} k_{B} T}\right)^{1-2 \rho} \\
& \times \frac{\left|\Gamma\left[\rho-j\left(e V / \pi k_{B} T\right)\right]\right|^{2}}{\Gamma(2 \rho)} \sinh \left(\frac{e V}{k_{B} T}\right),
\end{aligned}
$$

where $\rho=R / R_{Q}, \Gamma$ is Gamma function and $\gamma \approx$ 0.577 Euler's constant. The perturbation theory result, Eqs. (8)-(9), is valid if $I_{\text {cot }} \ll \rho I_{c}^{\mathrm{tr}}$. The latter condition means that the cotunneling events are rare and incoherent and this is nicely obeyed in our experiment.

Expression (9) pretty well gives the transport and gate voltage dependencies as well as the magnitude of the measured current (see calculated curves in Fig. 1). The values of the fitting parameters $R$ and $E_{J}$ are in good agreement with their estimated values $(10 \%$ and $13 \%$ deviation, respectively), while the effective temperature $T_{\text {eff }}$ of the resistor was found to be $90 \mathrm{mK}$ (cf. bath temperature of $20 \mathrm{mK}$ ). This value matches with $T_{\text {eff }}=70-80 \mathrm{mK}$ found in the same setup in measurements of the three-junction Al-Cr single electron pump with local resistors [17] and can be ascribed mostly to a noise effect.

We achieved the CTCP regime in the range of the gate charge $\left|Q_{0}\right| \leq \frac{e}{2}$. The gate modulation depth of cotunneling current was found to be $d \equiv I_{\cot }\left(\frac{e}{2}\right) / I_{\cot }(0) \approx 2$, 
that is in agreement with the value given by Eq. (5), i.e. $d=\left[I_{c}^{\operatorname{tr}}\left(\frac{e}{2}\right) / I_{c}^{\operatorname{tr}}(0)\right]^{2}=\frac{16}{9} \approx 1.78[18$. Approaching the points $Q_{0}= \pm e$ one could expect an increase in the cotunneling current by a factor of $\left(2 / \lambda_{0}\right)^{2} \approx 10^{2}$, but in these points STCP dominates because effective dissipation for tunneling across either junction is notably smaller than that for CTCP, $\rho \rightarrow \rho / 4$ [4]. As a manifestation of such behavior in our samples, we observed a current increase by more than two orders of magnitude when we applied a fast $(\sim 1 \mathrm{MHz})$ gate voltage sweep of amplitude $>e / C_{g}$. Such sweep allowed quasiparticle poisoning of the island to be avoided for short time intervals when passing the regions $Q_{0} \approx \pm e$.

Finally, an issue of particular interest is the suppression of CTCP at larger dissipation, i.e. at $R>R_{Q}[19$. The increase in $R$ should lead to a blockade behavior of the $I-V$ curves at small $V$ and development of a broad current peak moving to $V=\frac{4 e}{C}$. The latter feature can hardly be clear observed in experiment because at such a large voltage other current components (quasiparticle and combined pair-quasiparticle tunneling) are significant [11]. On the other hand, for small voltages between $k_{B} T / 2 e$ and $e / C$, cotunneling still dominates and for its estimation one can apply the zero temperature expansion of function $P[16$ and will get

$$
I_{\mathrm{cot}}=I_{0}\left(\frac{V}{V_{0}}\right)^{2 \rho-1}, \text { where } I_{0}=\frac{\pi \rho e^{-2 \gamma} \Phi_{0}\left(I_{c}^{\mathrm{tr}}\right)^{2}}{32 \Gamma(2 \rho) E_{c}}
$$

and $V_{0}=\frac{2 e}{\pi \rho C}$. Thus, the CTCP current obeys the power law, i.e. it is efficiently suppressed at large $\rho$. This effect was demonstrated and the results are presented in Fig. 2. Although the experimental voltage range was rather limited, the data are in good agreement with the dependence Eq. (10) for parameter values close to those estimated from measurements.

In conclusion, the obtained results on measurements of CTCP are also of practical importance, e.g., for improving the performance characteristics of the Cooper pair pumps. In the latter devices, CTCP may significantly degrade the accuracy of charge pumping [20]. In contrast to the normal junction case, an increase in the number $N$ of junctions in the superconducting pump array seems to be not suitable for suppressing unwanted CTCP [21]. On the other hand, the use of local resistors [17, 22] ensures such suppression and, therefore, is promising for the construction of an accurate Cooper pair pump with a reasonable number $(N=3-5)$ of junctions in the array.

This work was partially supported by the EU through the COUNT and SQUBIT-2 projects.

[1] Y. Nakamura, Yu. A. Pashkin and J. S. Tsai, Nature 398, 768 (1999); D. Vion, A. Aassime, A. Cottet, P. Joyez,
H. Pothier, C. Urbina, D. Esteve and M. H. Devoret, Science 296, 886 (2002).

[2] A. B. Zorin, S. V. Lotkhov, Yu. A. Pashkin, H. Zangerle, V. A. Krupenin, T. Weimann, H. Scherer and J. Niemeyer, J. Supercond. 12, 747 (1999).

[3] J. Delahaye, J. Hassel, R. Lindell, M. Sillanpää, M. Paalanen, H. Seppä and P. Hakonen, Science 299, 1045 (2003).

[4] F. K. Wilhelm, G. Schön and G. T. Zimányi, Phys. Rev. Lett. 87, 136802 (2001).

[5] J. B. Kycia, J. Chen, R. Therrien, Ç. Kurdak, K. L. Campman, A. C. Gossard and J. Clarke, Phys. Rev. Lett. 87, 017002 (2002).

[6] W. Lu, A. J. Rimberg and K. D. Maranowski, Appl. Phys. Lett. 81, 4976 (2002) and references therein.

[7] According to the classification introduced for single electron cotunneling by D. V. Averin and Yu. V. Nazarov, Phys. Rev. Lett. 65, 2446 (1990), CTCP belongs to the category of "elastic cotunneling," because it doesn't lead to excitation in the island.

[8] A. Amar, D. Song, C. J. Lobb and F. C. Wellstood, Phys. Rev. Lett. 72, 3234 (1994).

[9] S. V. Lotkhov, S. A. Bogoslovsky, A. B. Zorin, and J. Niemeyer, in International Workshop on Superconducting Nano-Electronics Devices, edited by J. Pekola et al., (Kluwer Academic/Plenum Publishers, New York, 2002), p. 105.

[10] D. V. Averin, Yu. V. Nazarov and A. A. Odintsov, Physica B 165\&166, 945 (1990).

[11] L. S. Kuzmin, Yu. V. Nazarov, D. B. Haviland, P. Delsing and T. Claeson, Phys. Rev. Lett. 67, 1161 (1991).

[12] G. L. Ingold, H. Grabert and U. Eberhardt, Phys. Rev. B 50, 395 (1994).

[13] K. K. Likharev and A. B. Zorin, J. Low Temp. Phys. 59, 347 (1985).

[14] A. B. Zorin, IEEE Trans. Instrum. and Meas. 46, 299 (1997).

[15] A. B. Zorin, Phys. Rev. Lett. 76, 4408 (1996).

[16] M. H. Devoret, D. Esteve, H. Grabert, G.-L. Ingold, H. Pothier and C. Urbina, Phys. Rev. Lett. 64, 1824 (1990).

[17] S. V. Lotkhov, S. A. Bogoslovsky, A. B. Zorin, and J. Niemeyer, Appl. Phys. Lett. 78, 946 (2001).

[18] Note that formula for the cotunneling rate presented in Ref. 4], i.e. $\Gamma_{\mathrm{CT}} \propto\left(\delta E_{\mathrm{ch}}\right)^{-2}$, gives the overestimated value of $d=\left[\delta E_{\mathrm{ch}}(0) / \delta E_{\mathrm{ch}}\left(\frac{e}{2}\right)\right]^{2}=4$.

[19] The suppression of single electron cotunneling by local resistors was studied by A. A. Odintsov, V. Bubanja and G. Schön, Phys. Rev. B 46, 6875 (1992); A. B. Zorin, S. V. Lotkhov, H. Zangerle and J. Niemeyer, J. Appl. Phys. 88, 2665 (2000).

[20] L. J. Geerligs, S. M. Verbrugh, P. Hadley, J. E. Mooij, H. Pothier, P. Lafarge, C. Urbina, D. Esteve and M. N. Devoret, Z. Phys. B: Condens. Matter 85, 349 (1991).

[21] J. P. Pekola, J. J. Toppari, M. Aunola, M. T. Savolainen and D. V. Averin, Phys. Rev. B 60, R9931 (1999).

[22] A. B. Zorin, S. A. Bogoslovsky, S. V. Lotkhov, and J. Niemeyer, in Macroscopic Quantum Coherence and Quantum Computing, edited by D. V. Averin et al., (Kluwer Academic/Plenum Publishers, New York, 2001), p. 147. 